\title{
Colossal magnetoresistance in manganites as a multicritical phenomenon
}

\author{
Shuichi Murakami ${ }^{1}$ and Naoto Nagaosa ${ }^{1,2}$ \\ ${ }^{1}$ Department of Applied Physics, University of Tokyo, Bunkyo-ku, Tokyo 113-8656, Japan \\ ${ }^{2}$ CERC, AIST Tsukuba Central 4, Tsukuba 305-8562, Japan
}

(Dated: October 26, 2018)

\begin{abstract}
The colossal magnetoresistance in manganites $\mathrm{AMnO}_{3}$ is studied from the viewpoint of multicritical phenomena. To understand the complicated interplay of various phases, we study the Ginzburg-Landau theory in terms of both the mean-field approximation and the renormalizationgroup analysis to compare with the observed phase diagram. Several novel features, such as the first-order ferromagnetic transition, and the dip in the transition temperature near the multicritical point, can be understood as driven by enhanced fluctuations near the multicritical point. Furthermore, we obtain a universal scaling relation for the $H / M-M^{2}$ plot (Arrott plot), which fits rather well with the experimental data, providing the further evidence for the enhanced fluctuation.
\end{abstract}

PACS numbers: 75.30.Vn 75.40.Cx 64.60.Kw 75.60.Ej

Colossal magnetoresistance (CMR) in manganites is one of the most dramatic phenomena shown by strongly correlated electronic systems, and extensive experimental studies have revealed many aspects of this effect [1, 2. However, its mechanism has been the subject of longstanding debates; many theories have been proposed such as double exchange [3], polaronic effect [4], and phase separation combined with percolation [5, 6] and Griffiths singularity [7]. Shown in Figs. 1 are the phase diagrams of the CMR manganites. They clearly evidence that the CMR is related to the concomitant antiferromagnetic $(\mathrm{AF})$ spin ordering, charge ordering $(\mathrm{CO})$, and orbital ordering $(\mathrm{OO})$ around the hole concentration $x=0.5$. Near the phase boundary between the $\mathrm{AF} / \mathrm{CO} / \mathrm{OO}$ and the ferromagnetic metallic (FM) state, the transition temperature has a sharp dip and the critical magnetic field $H_{c}$ is reduced considerably. Hence, the CMR is collective in nature and differs from single particle properties such as the transition from small to large polarons.

One of the subtle issues is the effect of the randomness


FIG. 1: Phase diagram for the manganites: (i) $\operatorname{Pr}_{0.55}\left(\mathrm{Ca}_{1-y} \mathrm{Sr}_{y}\right)_{0.45} \mathrm{MnO}_{3}$ \& and (ii) $\left(\mathrm{Nd}_{1-y} \mathrm{Sm}_{y}\right)_{0.55} \mathrm{Sr}_{0.45} \mathrm{MnO}_{3}$ [9]. Solid squares represent a first-order transition. Red dots represent the data used in the scaling analysis in the text. The red region at $0 \mathrm{~K}<T<50 \mathrm{~K}$ in (ii) is a spin-glass phase. on the CMR. The ramdomness induced by the alloying and/or the nonuniform strain affects physical quantities near the boundary of the two phases, and alters the critical properties easily. Figures 11 show the two systems (i) $\mathrm{Pr}_{0.55}\left(\mathrm{Ca}_{1-y} \mathrm{Sr}_{y}\right)_{0.45} \mathrm{MnO}_{3}$ and (ii) $\left(\mathrm{Nd}_{1-y} \mathrm{Sm}_{y}\right)_{0.55}$ $\mathrm{Sr}_{0.45} \mathrm{MnO}_{3}$. It is not trivial which system is more disordered, namely alloying (i) alkaline-earth atoms or (ii) rare-earth atoms. There are, however, three reasons to believe that the system (i) shows more intrinsic properties than (ii) which is mostly dominated by the disorder effect. The first is that the strong suppression of the ferromagnetic transition temperature and the appearance of the spin-glass type state in (ii) is well reproduced by recent model calculation in [6]. The second is that the phase diagrams in ordered and disordered half-doped manganites $L n_{1 / 2} \mathrm{Ba}_{1 / 2} \mathrm{MnO}_{3}$ [10, 11] closely resemble Fig. 1 (i) and (ii), respectively. The last one is that the scaling fit works almost perfectly in (i), while it does only in the limited region in (ii), as we show in this Letter. In (ii), the region near the phase boundary is dominated by the disorder effect. An appealing scenario for this behavior is the phase separation and/or percolation of the conducting paths [6]. This assumes a mixture of metallic and insulating domains; by applying magnetic field the metallic domains expand to result in the CMR. This is the static picture of the resistance network model controlled by the magnetic field. However, experiments on diffuse $\mathrm{X}$-ray scattering and Raman scattering revealed that intrinsic fluctuation is dynamic in nature [1]. Therefore, we need to take into account thermal and/or quantum fluctuations near the phase boundary. It is well-known that fluctuation is enhanced near a multicritical point, where more than two orders collide with each other. In this Letter we propose a new scenario based on this idea that the CMR originates from the enhanced fluctuation near the multicritical point, which is controlled by the small external magnetic field. We construct the phenomenological Ginzburg-Landau (GL) model based on symmetry argument, and next give a renormalization-group (RG) 
analysis for the multicritical phenomena to compare with experiments. This picture explains the scaling law and the first-order ferromagnetic phase transition as well as the enhanced sensitivity to the external magnetic field near the phase boundary.

The ordering pattern of $\mathrm{AF} / \mathrm{CO} / \mathrm{OO}$ is complicated with an enlarged unit cell. Several microscopic models for this state have been proposed [12. We employ here instead the phenomenological GL theory. We classify possible terms in the free energy functional according to the symmetry of the order parameters. The relevant order parameters are those of ferromagnetism $\vec{M}$, antiferromagnetism $\vec{S}$, charge ordering $\rho$, and orbital ordering $\vec{T}$. Here, we discuss the dimensionality of each order parameter. Both $\vec{M}$ and $\vec{S}$ are three-dimensional, while $\rho$ is scalar. The orbital pseudo-vector $\vec{T}$ is originally threedimensional but in the presence of the Jahn-Teller interaction, which prefers real linear combinations of the two wavefunctions $x^{2}-y^{2}$ and $3 z^{2}-r^{2}$, it should be regarded as two-dimensional: $\vec{T}=\left(T_{x}, T_{z}\right)$. The free-energy functional should be rotationally invariant in the spin space, but not in the orbital pseudo-spin space. Hence, $\vec{M}$ and $\vec{S}$ should appear in the form of $\vec{M}^{2}$ and $\vec{S}^{2}$. In contrast, third-order terms in $\vec{T}$ 's are allowed, because $-\vec{T}$ is not iquivalent to $-\vec{T}$. Linear terms in $\vec{T}$ 's are generated by the coupling to the external uniaxial strain field.

Next we consider the wavenumbers of the order parameters. From the spatial pattern of the $\mathrm{AF} / \mathrm{CO} / \mathrm{OO}$, we can easily see that wavenumber of each order is the following, $\rho:(\pi, \pi, 0), \vec{S}:(\pi, 0, \pi),(0, \pi, \pi)$, $(\pi / 2,-\pi / 2, \pi), \quad(-\pi / 2, \pi / 2, \pi), \vec{T}: \quad(0,0,0), \quad(\pi, \pi, 0)$, $(\pi / 2, \pi / 2,0),(-\pi / 2,-\pi / 2,0), \vec{M}:(0,0,0)$. Therefore, the allowed terms in the GL functional are

$$
\begin{aligned}
& F=\frac{1}{2} \int d^{3} r\left[(\nabla \vec{M})^{2}+(\nabla \vec{S})^{2}+(\nabla \vec{T})^{2}+(\nabla \rho)^{2}\right. \\
& +r_{M}(\vec{M})^{2}+r_{S}(\vec{S})^{2}+r_{T}(\vec{T})^{2}+r_{\rho}(\rho)^{2} \\
& +\left(r_{\rho T}^{x} T_{x}+r_{\rho T}^{z} T_{z}\right) \rho+g_{\rho S} \rho \vec{S}^{2}+g_{T} \operatorname{Re}\left(T_{x}+i T_{z}\right)^{3} \\
& \left.+u_{M}\left((\vec{M})^{2}\right)^{2}+u_{S}\left((\vec{S})^{2}\right)^{2}+u_{T}\left((\vec{T})^{2}\right)^{2}+u_{\rho} \rho^{4}\right] .(1)
\end{aligned}
$$

Here some remarks are in order. First, the bilinear coupling between $\rho$ and $\vec{T}$ enforces that these two orders accompany each other. This agrees with experiments. Second, the third-order term in $\vec{T}$ make the transition first-order. However the magnitude of the jump at the transition depends on the relative values of $r_{\rho}$ and $r_{T}$; when $r_{\rho} \ll r_{T}$, it becomes nearly second-order, while it is strongly first-order in the other limit. Experimentally, the $\mathrm{CO} / \mathrm{OO}$ transition in the narrow band-width side is nearly second-order, which means that the transition is mainly driven by the CO. Hence, we neglect below the orbital ordering $\vec{T}$. Third, the term $\rho \vec{S}^{2}$ prohibits the AF without the $\mathrm{CO}$, namely the $\mathrm{CO}$ occurs at a temperature higher than or as high as the AF. This is a generic con-
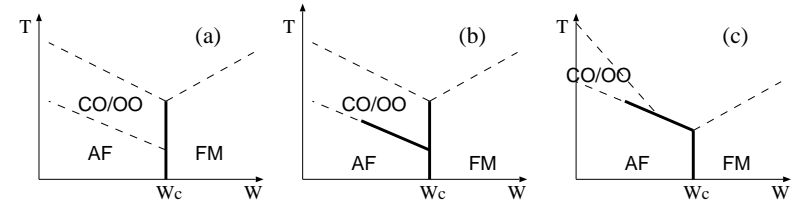

FIG. 2: Mean-field phase diagrams for the GL functional (11) for in the $W-T$ plane. Here $W$ is the bandwidth and $T$ is the temperature. The broken (solid) lines represent the second (first)-order phase transitions.

clusion from the symmetry independent of microscopic mechanisms, and is consistent with the experiments.

Figure 2 shows three possible mean-field phase diagrams for the GL functional (11), in the plane of the band-width $W$ and the temperature $T$. Figure $2(\mathrm{a})$ is the most relevant to the experimental results. Nonetheless, this mean-field analysis cannot capture several experimental features. One is the first-order FM transition in the wide-bandwidth side. Another is the dip of the transition temperatures of $\mathrm{FM}$ and $\mathrm{CO} / \mathrm{OO}$ near the critical bandwidth $W_{c}$. These two features are due to the fluctuations enhanced near $W_{c}$. This is the so-called multicritical phenomenon between the competing orders.

We now turn to the RG analysis of this fluctuation. Let us consider a system with competing two orders " $\mathrm{A}$ " and "B". We express their order parameters as an $N_{A^{-}}$ dimensional vector $\vec{\phi}_{A}$ and an $N_{B}$-dimensional vector $\vec{\phi}_{B}$. Assuming a rotational invariance in $\vec{\phi}_{A}$ and in $\vec{\phi}_{B}$, we can write its classical free energy up to quartic order as

$$
F_{\mathrm{cl}}=\frac{1}{2}\left(r_{A} \phi_{A}^{2}+r_{B} \phi_{B}^{2}\right)+\frac{1}{4}\left(u \phi_{A}^{4}+2 w \phi_{A}^{2} \phi_{B}^{2}+v \phi_{B}^{4}\right) .
$$

In the present case, the phases $\mathrm{A}$ and $\mathrm{B}$ correspond to the FM phase and the $\mathrm{CO} / \mathrm{OO}$ phase. Thus, $\phi_{A}$ is a magnetization $\vec{M}$. The average value $\frac{N_{A} r_{A}+N_{B} r_{B}}{N_{A}+N_{B}}$ is roughly proportional to the temperature measured from the multicritical point, while $r_{A}-r_{B}$ is proportional to the deviation of bandwidth from the multicritical point. The model (2) was studied in 13 by the RG. The fluctuationinduced first-order transition 14 is known to occur in (2) when $w>\sqrt{u v}$ [15]. For an understanding of the fluctuation-induced first-order transition, an effective potential [16] is a useful tool. The effective potential is given by adding fluctuation contribution to the classical Lagrangian. Bare coefficients in the effective potential should be renormalized to remove ultraviolet divergence at $d=4$. By adding counterterms as in [16], we get

$$
\begin{aligned}
F & =\frac{1}{2}\left(r_{A} \phi_{A}^{2}+r_{B} \phi_{B}^{2}\right)+\frac{1}{4}\left(u \phi_{A}^{4}+2 w \phi_{A}^{2} \phi_{B}^{2}+v \phi_{B}^{4}\right) \\
& +\left(N_{A}-1\right) \frac{\alpha_{A}^{2}}{8}\left(\ln \frac{\alpha_{A}}{\kappa^{2}}+\frac{1}{2}\right)+\frac{\gamma_{+}^{2}}{8}\left(\ln \frac{\gamma_{+}}{\kappa^{2}}+\frac{1}{2}\right) \\
& +\left(N_{B}-1\right) \frac{\alpha_{B}^{2}}{8}\left(\ln \frac{\alpha_{B}}{\kappa^{2}}+\frac{1}{2}\right)+\frac{\gamma_{-}^{2}}{8}\left(\ln \frac{\gamma_{-}}{\kappa^{2}}+\frac{1}{2}\right)(3)
\end{aligned}
$$


where

$$
\begin{aligned}
& \left(\begin{array}{c}
\alpha_{A} \\
\alpha_{B}
\end{array}\right)=\left(\begin{array}{c}
r_{A} \\
r_{B}
\end{array}\right)+\left(\begin{array}{cc}
u & w \\
w & v
\end{array}\right)\left(\begin{array}{c}
\phi_{A}^{2} \\
\phi_{B}^{2}
\end{array}\right) \\
& \left(\begin{array}{c}
\beta_{A} \\
\beta_{B}
\end{array}\right)=\left(\begin{array}{c}
r_{A} \\
r_{B}
\end{array}\right)+\left(\begin{array}{cc}
3 u & w \\
w & 3 v
\end{array}\right)\left(\begin{array}{c}
\phi_{A}^{2} \\
\phi_{B}^{2}
\end{array}\right) \\
& \gamma_{+}+\gamma_{-}=\beta_{A}+\beta_{B}, \quad \gamma_{+} \gamma_{-}=\beta_{A} \beta_{B}-4 w \phi_{A}^{2} \phi_{B}^{2},
\end{aligned}
$$

and $\kappa$ is a parameter setting a scale of momentum. We neglected the terms higher than $O(4-d)$. The quantities $r_{i}, u, v, w$ in (3) are renormalized ones and are finite.

Let us consider the fluctuation-induced first-order transition 114 between the disordered phase and the "A" phase. We thus assume that the "B" field $\phi_{B}$ is not ordered, i.e. $\phi_{B}=0$. In the RG language, the fluctuationinduced first-order transition occurs when the RG flow runs into an unstable region of the model (2). It means that sixth-order terms, though omitted in (2), are necessary for stability, leading to a first-order transition. Hence, if the RG flow crosses the boundary of the stability region, $u=0$, the system undergoes a first-order transition to the "A" phase. In other words, we follow the RG flow for $u, v, w$ 13 until we reach the line $u=0$. Let $\kappa_{1}$ denote the value of $\kappa$ when $u(\kappa)=0$. Other quantities as $r_{A}, r_{B}, \phi_{A}$ are also renormalized. It is worth noting that these quantities are renormalized multiplicatively, i.e. $r_{i}\left(\kappa_{1}\right)=r_{i} f\left(\kappa_{1}\right), \phi_{A}\left(\kappa_{1}\right)=\phi_{A} g\left(\kappa_{1}\right)$, where $r_{i}$ and $\phi_{A}$ are the initial values. Thus, renormalization is merely a change of scale for them. The condition $u\left(\kappa_{1}\right)=0$ greatly simplifies the above free energy as

$$
F=\frac{1}{2} r_{A} \phi_{A}^{2}+N_{A} f\left(r_{A}\right)+N_{B} f\left(r_{B}+w \phi_{A}^{2}\right),
$$

where $f(x)=\frac{x^{2}}{8}\left(\ln x+\frac{1}{2}\right)$ and $\kappa_{1}$ is set as unity since it can be absorbed by the change of scale of other variables. The equation of state is

$$
H_{A}=\frac{\partial F}{\partial \phi_{A}}=r_{A} \phi_{A}+2 \phi_{A} N_{B} w f^{\prime}\left(r_{B}+w \phi_{A}^{2}\right) .
$$

where $H_{A}$ is a field conjugate to the $\phi_{A}$ field. Since we identify this $\phi_{A}$ as a magnetization $\vec{M}, H_{A}$ is a magnetic field $H$. It is convenient to rewrite (8) as

$$
\frac{H}{M}=r_{A}+2 w N_{B} f^{\prime}\left(r_{B}+w M^{2}\right) .
$$

Therefore if we plot $H / M$ versus $M^{2}$ at various temperature and bandwidth, all the plots will be degenerate. In other words, the $H / M-M^{2}$ curve, called the Arrott plot, will undergo parallel transport when we change temperature or bandwidth. One would notice that in a certain range of temperature and bandwidth this universal curve crosses the horizontal axis once or twice. In that case some part of the universal curve becomes unphysical, and the system undergoes a first-order phase transition by increasing the magnetic field. The system is either ferromagnetic or metamagnetic.
To verify this scenario, we used two series of data in Figs. 证: (i) for $y=0.25,0.3,0.4$ and (ii) for $0.1 \leq y \leq 0.4$, with varying $y$ and $T$. We can regard $y$ as a parameter controlling the bandwidth. We expect that $r_{A}$ and $r_{B}$ are functions of $y$ and $T$ while $w$ is a constant. We expand $r_{A}$ and $r_{B}$ in the vicinity of the multicritical point as $r_{A}=c_{A T} \Delta T+c_{A y} \Delta y, r_{B}=c_{B T} \Delta T+c_{B y} \Delta y$, where $c_{A T}, c_{B T}, c_{A y}, c_{B y}$ are constants. In view of (9), $c_{A T}, c_{B T}$ $\left(c_{A y}, c_{B y}\right)$ represents an amount of parallel displacement of the $H / M-M^{2}$ plot when the temperature $T$ (doping $y$ ) is changed. We fitted the data in the following way. First we varied $c_{A T}, c_{B T}, c_{A y}, c_{B y}$ so that the plots for various $y$ and $T$ overlap most after the parallel displacement. Then the scales of the abscissa and the ordinate are varied to fit to (9). We used the data shown as red dots in Figs. 11. We discarded the data with $M>1.6 \mu_{\mathrm{B}}$, because when the magnetization approaches the saturation value, the GL functional up to quadratic order is no longer appropriate. We also discarded the data for $0.5 \leq y \leq 0.8$ in (ii). They do not fit well with the scaling curve. This is reasonable because in (ii) critical fluctuations are washed out near the multicritical point, by which the scaling plot deviates from (9).

The resulting plot is shown in Fig. 3. The plots fall into one curve with good accuracy. This shows that the fluctuation is enhanced near the multicritical point. Because the critical scaling (9) holds for a wide range of data shown in Fig. 3, the critical region is rather large $(\sim 80 \mathrm{~K})$. It manifests strong correlation of electrons in the manganites. Whether the system is critical or not is determined by $\xi$, measured by a length scale $\xi_{0} \sim \frac{E_{\mathrm{F}}}{\Delta} a$, where $\Delta$ is a gap, $a$ is a lattice constant, and $E_{\mathrm{F}}$ is the Fermi energy. In the manganites $\Delta \sim E_{\mathrm{F}}$ yields $\xi_{0} \sim a$. Temperature dependence of $\xi$ in a related compound $\mathrm{Pr}_{0.5} \mathrm{Ca}_{0.5} \mathrm{MnO}_{3}$ can be obtained from Fig. 2(c) of [17]. It is $\xi \sim 100 \AA$, near the transition temperature $T_{c}=235 \mathrm{~K}$, and is $\xi \sim 20 \AA$ even at $300 \mathrm{~K}$, which is $65 \mathrm{~K}$ higher than $T_{c}$. Therefore, $\xi$ is much longer that $\xi_{0}$ in the wide temperature range, implying that the critical region should be large.

The enhanced fluctuation makes the system sensitive to the external magnetic field $H$. The $\mathrm{CO} / \mathrm{OO}$ state easily becomes the FM state by a weak magnetic field. Let $H_{c}$ denote this critical field. An exponent $x$ defined by $H_{c} \propto\left(W_{c}-W\right)^{x}$ represents the sensitivity to the external field. Without the fluctuation, $x$ should be equal to unity. On the other hand, in the multicritical region but not in the fluctuation-induced first-order-transition region, $x$ is controlled by the bicritical fixed point, and is larger than unity. Calculation based on the $(4-\epsilon)$ expansion up to $O\left(\epsilon^{2}\right)$ results in $x=\frac{\beta \delta}{\phi} \sim 1.37$, where $\phi$ is the crossover exponent. It can be compared with the experimental value $x \sim 1.6$, extracted from the data for $\mathrm{Pr}_{0.65}\left(\mathrm{Ca}_{1-y} \mathrm{Sr}_{y}\right)_{0.35} \mathrm{MnO}_{3}$ [18]. This increase of $x$ near the multicritical point, i.e. sensitivity to the external field, emerges as the CMR phenomena. 


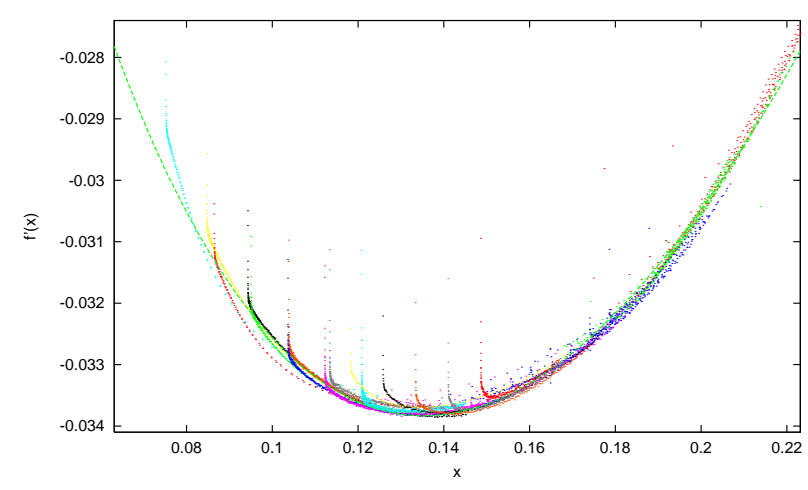

FIG. 3: Scaling plot for (i) $\operatorname{Pr}_{0.55}\left(\mathrm{Ca}_{1-y} \mathrm{Sr}_{y}\right)_{0.45} \mathrm{MnO}_{3}$ and (ii) $\left(\mathrm{Nd}_{1-y} \mathrm{Sm}_{y}\right)_{0.55} \mathrm{Sr}_{0.45} \mathrm{MnO}_{3}$. The broken line represent the $f^{\prime}(x)=\frac{x}{4}(\ln x+1)$ plot. Each dot show the data for fixed $T$ and $y$.

Here we discuss the effect of disorder, and the relevance of the Griffiths singularity [7] to the present study. It has been pointed out that in the temperature region $T_{c}<T<T_{c}^{(0)}$ where $T_{c}^{(0)}$ is the transition temperature for the pure system while $T_{c}$ is the suppressed one by the random dilution, there appears some singularity in the time-dependence of correlation functions of the order parameter, such as the extended power-law decay. However, this singularity does not occur in the thermodynamic quantities which we discussed in this Letter. It is possible that the fixed point for the pure system is unstable against disorder, but according to the Harris criterion the bicritical fixed point described above is at least locally stable [16]. Hence, the thermodynamic properties are described by the usual bicritical fixed point, although the location of the critical point is shifted. However, the disorder effect on the first-order phase transition are beyond the scope of the present work, and ref. [6] is relevant to this issue. The percolation scenario could also lead to the CMR, where the resistivity will depend sensitively on random realization of the metallic paths etc., and consequently on samples. This behavior has been actually observed in dilutely Cr-doped manganites 19 . $\mathrm{Cr}$ ions destroy the $\mathrm{CO} / \mathrm{OO}$ locally, and introduce the FM region. In these dilutely doped samples, the resistivity at low temperature depends on the Cr-concentration dramatically, and the hysteresis appears in the temperature cycle. In contrast, when the Cr-concentration increases, the resisivity no longer depends on samples or heat cycle. In the latter case, the thermodynamic phases are well-defined and the CMR is triggered by the phase change between them, which is the subject of our study here. Hence, there are two types of the CMR; one is due to the percolating path and the other is due to the multicritical fluctuation near the phase change. In the zero temperature limit, dynamics and statics are coupled and the Griffiths singularities influence the whole quantum critical phenomena. In the manganites, this possibility seems to be prevented by the glassy state appearing at low temperatures.

We would like to acknowledge Y. Tokura, Y. Tomioka, E. Dagotto, and H. Kageyama for fruitful discussions. This work is supported by Grant-in-Aids from the Ministry of Education, Culture, Sports, Science, and Technology.

[1] Y. Tokura, N. Nagaosa, Science 288, 462 (2000) and references therein.

[2] E. Dagotto, T. Hotta, A. Moreo, Phys. Rep. 344, 1 (2001).

[3] N. Furukawa, J. Phys. Soc. Jpn. 63, 3214 (1994).

[4] A. J. Millis, P. B. Littlewood, and B. I. Shraiman, Phys. Rev. Lett. 74, 5144 (1995).

[5] S. Mori, C. H. Chen, S-W. Cheong, Phys. Rev. Lett. 81, 3972 (1998); Y. Moritomo, Phys. Rev. B60, 10374 (1999); M. Uehara et al., Nature 399, 560 (1999).

[6] A. Moreo, S. Yunoki, E. Dagotto, Science 283, 2034 (1999); J. Burgy et al., Phys. Rev. Lett. 87, 277202 (2001).

[7] M. B. Salamon, P. Lin, and S. H. Chun, Phys. Rev. Lett. 88, 197203 (2002)

[8] T. Tomioka and Y. Tokura, preprint, submitted to Phys. Rev. B.

[9] T. Tomioka and Y. Tokura, preprint.

[10] D. Akahoshi et al., preprint.

[11] H. Kageyama et al., preprint; T. Nakajima et al., preprint (cond-mat/0207410).

[12] J. van den Brink, G. Khaliullin, and D. Khomskii Phys. Rev. Lett. 83, 5118 (1999); I. V. Solovyev and K. Terakura Phys. Rev. Lett. 83, 2825 (1999).

[13] D. R. Nelson, J. M. Kosterlitz, and M. E. Fisher, Phys. Rev. Lett. 33, 813 (1974); J. M. Kosterlitz, D. R. Nelson, and M. E. Fisher, Phys. Rev. B13, 412 (1974).

[14] D. J. Wallace, J. Phys. C6, 1390 (1973); T. Natterman and S. Trimper, J. Phys. A8, 2000 (1975); S. A. Brazovskii and I. E. Dzyaloshinskii, JETP Lett. 21, 164 (1975); J. Rudnick, Phys. Rev. B18, 1406(1978); E. Domany, D. Mukamel, and M. E. Fisher, Phys. Rev. B15, 5432 (1977).

[15] S. Murakami and N. Nagaosa, J. Phys. Soc. Jpn. 692395 (2000).

[16] D. J. Amit, Field Theory, the Renormalization Group and Critical Phenomena (World Scientific, Singapore, 1993) and references therein; H. H. Iacobson and D. J. Amit, Ann. Phys. 133, 57(1981).

[17] S. Shimomura et al., Phys. Rev. B62, 3875 (2000).

[18] Y. Tomioka et al., J. Phys. Soc. Jpn. 66, 302 (1997).

[19] B. Raveau, A. Maignan, C. Martin, J. Solid State Chem. 130, 162 (1997); T. Kimura et al., Phys. Rev. Lett. 83, 3940 (1999). 